\title{
Miocardiopatía periparto en 2021: ¿con qué contamos para su tratamiento?
}

\author{
Peripartum cardiomyopathy in 2021: what is available for its management? \\ Luis Gutiérrez-Abarca* \\ Cardiología Clínica, Centro Cardiovascular Regional Centro Occidental ASCARDIO, Barquisimeto, Venezuela
}

\begin{abstract}
Resumen
Introducción: La miocardiopatía periparto (MCPP) es una condición que afecta a mujeres al final del embarazo o durante los primeros meses del periodo puerperal, caracterizada por insuficiencia cardiaca secundaria a disfunción sistólica del ventrículo izquierdo de origen idiopático; por lo tanto, esta se considera un diagnóstico de exclusión. El manejo de la MCPP resulta ser un reto que requiere un abordaje multidisciplinario que debe incluir, como mínimo, al cardiólogo y al obstetra, ya que existen especiales consideraciones debido a las precauciones que deben tomarse en cuenta para conservar la salud fetal y materna. Objetivo: Realizar una revisión sobre la evidencia científica disponible hasta la fecha en relación con el tratamiento de la MCPP. Método: Se realizó una revisión sistemática de la literatura en la base de datos PubMed bajo los siguientes términos: peripartum cardiomyopathy, pregnancy and heart failure y pregnancy and cardiomyopathy. Se seleccionaron artículos sin restricción de idioma ni fecha de publicación. Conclusiones: Ha habido un incremento en el conocimiento de ciertos aspectos sobre la MCPP en los últimos años; sin embargo, queda mucho por conocer con respecto a sus mecanismos patogénicos. Si bien la bromocriptina parece ofrecer buenos resultados, es necesaria mayor investigación para averiguar su verdadero rol en esta condición. Deben realizarse más estudios para evaluar los posibles beneficios y la seguridad de las nuevas terapias propuestas.
\end{abstract}

Palabras clave: Miocardiopatía periparto. Insuficiencia cardiaca. Embarazo.

\begin{abstract}
Introduction: Peripartum cardiomyopathy (PPCM) is a condition that affects women towards the end of pregnancy or during the first months after delivery characterized by heart failure secondary to left ventricle systolic dysfunction of idiopathic origin, thus, it is considered as an exclusion diagnosis. PPCM management is a challenge that requires a multidisciplinary approach that must include at least the cardiologist and the obstetrician because of the precautions that must be considered to preserve fetal and maternal health. Objective: To perform a review on the evidence available to date regarding the treatment of PPCM. Method: A systematic review was performed on the PubMed database using the following terms: peripartum cardiomyopathy, pregnancy and heart failure y pregnancy and cardiomyopathy. Articles were selected with no language or publication date restrictions. Conclusions: An increase in awareness of PPCM has occurred during the last few years, however, there is still much to discover about its pathogenic mechanisms. Bromocriptine therapy seems to offer goods results in these patients, further research is needed in order to know its true role in this condition. Other proposed therapies are yet to be studied to know their possible benefits and safety profile.
\end{abstract}

Key words: Peripartum cardiomyopathy. Heart failure. Pregnancy.

\section{Correspondencia:}

*Luis Gutiérrez-Abarca

E-mail: drluisgutierrezab@gmail.com
Disponible en internet: 07-01-2022

Rev Colomb Cardiol. 2021;28(6):523-529 www.rccardiologia.com 0120-5633 / @ 2021 Sociedad Colombiana de Cardiología y Cirugía Cardiovascular. Publicado por Permanyer. Este es un artículo open access bajo la licencia CC BY-NC-ND (http://creativecommons.org/licenses/by-nc-nd/4.0/). 


\section{Introducción}

La miocardiopatía periparto (MCPP) es una condición que afecta a mujeres al final del embarazo o durante los primeros meses del periodo puerperal, caracterizada por producir insuficiencia cardiaca (IC) secundaria a disfunción sistólica del ventrículo izquierdo (VI) de origen idiopático; por lo tanto, se considera un diagnóstico de exclusión. Según el grupo de trabajo de MCPP de la Sociedad Europea de Cardiología (SEC), se define como una «miocardiopatía que se presenta con insuficiencia cardiaca secundaria a disfunción sistólica del VI hacia el final del embarazo o en los meses posteriores al parto en ausencia de otras causas de insuficiencia cardiaca» ${ }^{1}$, siendo esta una definición más amplia que la propuesta inicialmente en la década de 1990 en los Estados Unidos de América por el National Heart, Lung, and Blood Institute, ya que esta incluía solo aquellos casos que ocurrían a partir del último mes del embarazo y durante el puerperio².

Desde el punto de vista epidemiológico, los datos relacionados con la incidencia de MCPP son muy variables en las distintas regiones del mundo, esto debido a una verdadera diferencia en la incidencia en zonas geográficas $y$, probablemente, al subregistro que puede existir en algunos países. Ejemplo claro de esta diferencia en la frecuencia de dicha condición es la alta incidencia reportada en algunos países de los continentes africano y asiático, en los cuales destaca Nigeria, donde se ha reportado una incidencia de 1 por cada 102 embarazos $^{3}$; por otra parte, en Haití se reporta 1 por cada 300 embarazos $^{4}$, y en China 1 por cada 350 embarazos $^{5}$. En contraste, hay reportes mucho menores en países como Japón, donde se reporta 1 caso por cada 20,000 embarazos ${ }^{6}$, y Dinamarca, con 1 por cada $10,000^{7}$. Esto sugiere la existencia de factores de predisposición genética, factores ambientales y su relación con otras patologías y condiciones de mayor prevalencia en ciertas poblaciones. La incidencia en diferentes regiones se resume en la tabla 1.

Si bien la MCPP es una condición con relativa baja incidencia en la población en comparación con otras afecciones del sistema cardiovascular, su carácter idiopático, su asociación con complicaciones cardiovasculares graves y las particularidades que implica la terapéutica durante el embarazo han despertado un gran interés por su estudio. Esta revisión resume parte de la información disponible hasta la fecha y su base en la evidencia científica sobre las tendencias actuales en el manejo de la MCPP.
Tabla 1. Incidencia de miocardiopatía periparto en diferentes lugares del mundo

\begin{tabular}{|l|c|}
\hline País/región & Incidencia (por nacidos vivos) \\
\hline Nigeria & $1 / 102$ \\
\hline Haití & $1 / 300$ \\
\hline China & $1 / 346$ \\
\hline Estados Unidos de América & $1 / 968$ \\
\hline Sudáfrica & $1 / 1000$ \\
\hline California, USA & $1 / 2066$ \\
\hline Malasia & $1 / 2941$ \\
\hline Suecia & $1 / 5719$ \\
\hline Dinamarca & $1 / 10,194$ \\
\hline Japón & $1 / 20,000$ \\
\hline
\end{tabular}

Adaptada de Honigberg y Givertz ${ }^{32}$.

El manejo de la MCPP resulta ser un reto que requiere un abordaje multidisciplinario que debe incluir, como mínimo, al cardiólogo y al obstetra, ya que existen especiales consideraciones debido a las precauciones que deben tomarse para conservar la salud fetal y materna, lo que diferencia esta condición de la IC en el resto de la población. Un factor importante del cual dependen las decisiones con respecto a la terapéutica es el momento en que se realiza el diagnóstico (embarazo o puerperio), tomando en cuenta la teratogenicidad de ciertos fármacos que suelen usarse en pacientes con IC, los efectos de la terapia farmacológica sobre la lactancia materna y, por otra parte, las decisiones relacionadas con la vía de obtención del producto de la gestación. La estrategia farmacológica recomendada en la actualidad puede ser resumida en cinco pilares fundamentales que se agrupan bajo el acrónimo BOARD (Bromocriptina, fármacos Orales para la IC, Anticoagulación, fármacos vasoRrelajantes y Diuréticos), el cual fue propuesto por Arrigo, et al..$^{8}$ en 2017 y probado con buenos resultados en Alemania; de acuerdo con esa experiencia, plantearon que estos cinco aspectos deben ser la base del tratamiento de estas pacientes según los datos provenientes de la evidencia.

\section{Bromocriptina}

Empleada inicialmente junto con la cabergolina como agente terapéutico para la supresión de la lactancia mediante la inhibición de la secreción de prolactina, su aprobación fue retirada para este uso en 1995 en los 
Estados Unidos de América por su asociación con complicaciones trombóticas, como infarto de miocardio e ictus $^{9}$. Años después, al realizarse algunas investigaciones que asociaron estrés oxidativo, la liberación de una forma de prolactina de $16 \mathrm{kDa}$ y otros factores con la ocurrencia de MCPP, se realizaron estudios experimentales en ratas a las cuales se indujo la secreción de esta proteína, que demostraron que la inhibición de la síntesis de prolactina mediante el uso de bromocriptina produjo resultados favorables en la evolución de la patología ${ }^{10}$. Posterior a esto se publicaron reportes de casos en humanos que demostraron efectos beneficiosos de su uso como terapia adicional al tratamiento convencional para la $\mathrm{IC}^{11,12}$. A raíz de lo anteriormente mencionado se han realizado hasta la fecha tres ensayos clínicos importantes sobre el uso de bromocriptina en pacientes con MCPP. El primero de ellos, realizado en Sudáfrica y publicado en 2010, incluyó 20 mujeres ingresadas por MCPP que fueron aleatorizadas para recibir terapia convencional para la IC o terapia convencional más bromocriptina a dosis de $2.5 \mathrm{mg}$ dos veces al día por 2 semanas, y luego una vez al día por 6 semanas. El grupo que recibió terapia convencional más bromocriptina tuvo mejor evolución clínica a los 6 meses, y todas las pacientes evolucionaron de clase funcional III-IV de la New York Heart Association, mientras que en el otro grupo todas se mantuvieron en clase funcional III-IV $(p=0.008)$; por otra parte, la fracción de eyección del ventrículo izquierdo (FEVI) incrementó a los 6 meses del $27 \pm 8 \%$ al $57 \pm 11 \%$, y en el otro grupo del $27 \pm 8 \%$ al $36 \pm 11 \%$ ( $p=0.007)$. Hubo un deceso en el grupo experimental y cuatro en el grupo control, y no se registraron complicaciones trombóti$\operatorname{cas}^{13}$. El segundo estudio, también realizado en África y publicado en 2017, con una metodología similar al anterior, aleatorizó 96 mujeres con MCPP para recibir terapia convencional o terapia convencional más bromocriptina $2,5 \mathrm{mg}$ dos veces al día por 4 semanas. A los 12 meses de seguimiento, el grupo experimental tuvo una FEVI en promedio del $53.9 \%$, frente al $45.9 \%$ en el grupo control $(p=0.001)$, y la excursión sistólica del anillo tricuspídeo fue de $22.7 \mathrm{~mm}$ y $20.9 \mathrm{~mm}$, respectivamente $(p=0.001)$. La mortalidad fue elevada, pero significativamente menor en el grupo que recibió bromocriptina: $17 \%$ frente a $29 \%(p=0.0001)^{14}$. El tercer estudio fue llevado a cabo en Alemania y se publicó en 2017, y en él se aleatorizó a 63 pacientes con MCPP y $\mathrm{FEVI}<35 \%$ para recibir terapia convencional más un régimen corto de bromocriptina ( $2.5 \mathrm{mg}$ por 1 semana) o un régimen extendido $(2.5 \mathrm{mg}$ dos veces al día por 2 semanas y $2.5 \mathrm{mg}$ por 6 semanas). No hubo diferencias significativas en el incremento de la FEVI ni en la recuperación completa definida como FEVI > $50 \%$. El grupo que recibió el régimen corto de bromocriptina presentó un incremento del $21 \pm 11 \%$ en la $\mathrm{FEVI}$, y el grupo que recibió el régimen extendido del $24 \pm 11 \%(p=0.381)$. El $52 \%$ de las pacientes en el grupo del régimen corto alcanzaron una $\mathrm{FEVI}>50 \%$, frente al $68 \%$ en el otro grupo $(p=0.651)$. Hubo tres eventos tromboembólicos en el grupo del régimen corto, y no hubo ningún deceso ${ }^{15}$.

Basándose en los hallazgos de estos ensayos, en la actualidad se recomienda el uso de bromocriptina para el tratamiento de la MCPP a dosis de $2.5 \mathrm{mg}$ al día durante 1 semana en casos no complicados y $2.5 \mathrm{mg}$ dos veces al día por 2 semanas, seguido de $2.5 \mathrm{mg}$ al día por 6 semanas, en casos con FEVI $<25 \%$ o shock cardiogénico, siempre acompañado de anticoagulación con heparina de bajo peso molecular o heparina no fraccionada, por su asociación con eventos tromboembólicos ${ }^{16}$. El uso de este fármaco sigue siendo considerado una alternativa experimental. Recientemente, el grupo Investigation in Pregnancy Associate Cardiomyopathy (IPAC) propuso la realización de un ensayo multicéntrico, aleatorizado y controlado con placebo, para evaluar el efecto de la bromocriptina en la recuperación miocárdica y sus desenlaces clínicos, bajo el nombre de REBIRTH (Randomized Evaluation of Bromocriptine In Myocardial Recovery Therapy), el cual se encuentra en planificación ${ }^{17}$.

\section{Fármacos convencionales para la insuficiencia cardíaca}

La terapia convencional de fármacos orales para la IC debe instaurarse tan pronto como las condiciones de la paciente lo permitan, tomando en cuenta el periodo en el cual se realice el diagnóstico por la contraindicación de algunos fármacos durante el embarazo debido a sus efectos teratogénicos, y otros durante el puerperio por ser excretados en la leche materna. La tabla 2 resume el estado de los diferentes fármacos para la IC en relación a su indicación o contraindicación en el embarazo o durante la lactancia. Se conoce con claridad que los inhibidores de la enzima convertidora de la angiotensina y los antagonistas de los receptores de la angiotensina II, y más recientemente los inhibidores de los receptores de angiotensina-neprilisina, tienen efectos teratogénicos, por lo que están contraindicados en el embarazo ${ }^{18,19}$. Durante el puerperio, algunos pueden ser usados con seguridad en pacientes que practican lactancia materna. Los betabloqueadores pueden usarse, tal como en la IC 
Tabla 2. Fármacos de tratamiento convencional para la insuficiencia cardiaca y anticoagulantes. Indicaciones y contraindicaciones en el embarazo y durante la lactancia

\begin{tabular}{|c|c|c|c|c|}
\hline Fármaco & Embarazo & Efectos adversos & Indicación & Lactancia \\
\hline \multicolumn{5}{|c|}{ Tratamiento convencional para IC } \\
\hline Diuréticos de ASA & Sí & $\begin{array}{l}\text { Hipovolemia-hipoperfusión } \\
\text { placentaria }\end{array}$ & $\begin{array}{l}\text { Signos y síntomas de } \\
\text { congestión }\end{array}$ & Si \\
\hline $\begin{array}{l}\text { Betabloqueadores } \\
\text { (metoprolol-bisoprolol) }\end{array}$ & Sí & $\begin{array}{l}\mathrm{RCIU} \text {, bradicardia fetal e } \\
\text { hipoglucemia }\end{array}$ & $\begin{array}{l}\text { Tratamiento convencional para } \\
\text { IC }\end{array}$ & Si \\
\hline Hidralazina-nitratos & Sí & Hipotensión & $\begin{array}{l}\text { Reducción de postcarga y } \\
\text { precarga }\end{array}$ & Si \\
\hline Digoxina & Sí & $\begin{array}{l}\text { No se han reportado efectos } \\
\text { adversos en fetos ni neonatos }\end{array}$ & IC - disfunción sistólica del VI & $\mathrm{Si}$ \\
\hline IECA/ARAII & No & $\begin{array}{l}\text { Anuria, oligoamnios, } \\
\text { teratogenicidad, RCIU, PCA, } \\
\text { muerte fetal }\end{array}$ & $\begin{array}{l}\text { Despues del parto como parte } \\
\text { del tratamiento convencional } \\
\text { para IC }\end{array}$ & $\mathrm{Si}$ \\
\hline ARM & No & $\begin{array}{l}\text { En ratas se ha asociado a } \\
\text { feminización y cambios en el } \\
\text { aparato reproductor }\end{array}$ & $\begin{array}{l}\text { Despues del parto como parte } \\
\text { del tratamiento convencional } \\
\text { para IC }\end{array}$ & Si. (Espironolactona) \\
\hline ARNI & No & Mismos que IECA/ARAII & $\begin{array}{l}\text { Despues del parto como parte } \\
\text { del tratamiento convencional } \\
\text { para IC }\end{array}$ & $\begin{array}{l}\text { No hay evidencia en } \\
\text { humanos }\end{array}$ \\
\hline Ivabradina & $\begin{array}{l}\text { Datos } \\
\text { insuficientes }\end{array}$ & Datos insuficientes & $\begin{array}{l}\text { Despues del parto como parte } \\
\text { del tratamiento convencional } \\
\text { para IC }\end{array}$ & $\begin{array}{l}\text { No hay evidencia en } \\
\text { humanos }\end{array}$ \\
\hline \multicolumn{5}{|c|}{ Anticoagulantes } \\
\hline HBPM & Sí & $\begin{array}{l}\text { Precaución al momento del } \\
\text { parto. No atraviesa la barrera } \\
\text { placentaria. Considerar } \\
\text { monitorización de niveles de } \\
\text { anti Xa }\end{array}$ & $\begin{array}{l}\text { Prevención y tratamiento de } \\
\text { complicaiones } \\
\text { tromboembólicas y puente } \\
\text { hacia warfarina en postparto }\end{array}$ & Sí \\
\hline Warfarina & No & $\begin{array}{l}\text { Embriopatía y fetopatía por } \\
\text { warfarina }\end{array}$ & $\begin{array}{l}\text { Prevención y tratamiento de } \\
\text { complicaiones } \\
\text { tromboembólicas }\end{array}$ & Sí \\
\hline ACOD & \multicolumn{4}{|c|}{ No hay datos sobre seguridad o eficacia durante embarazo ni lactancia } \\
\hline Leyenda & Seguro & Contraindicado & No existe evidencia & \\
\hline
\end{tabular}

ACOD: anticoagulantes orales directos; ARAll: Antagonistas de los receptores de angiotensina II; ARM: Antagonistas de los receptores de mineralocorticoides; ARNI: antagonistas de los receptores de neprilisina; HBPM: heparinas de bajo peso molecular; IC: insuficiencia cardíaca; IECA: inhibidores de la enzima convertidora de angiotensina; PCA: persistencia del conducto arterioso; RCIU: restricción del crecimiento intrauterino; VI: ventrículo izquierdo.

Adaptada de Davis, et al. ${ }^{7}$

en el resto de la población, iniciándose de forma cuidadosa según sea el estado hemodinámico, y titularse hasta la dosis máxima tolerada. Se consideran seguros los cardioselectivos por no tener efecto sobre la musculatura uterina; entre estos se prefieren el metoprolol y el bisoprolol por asociarse con menor frecuencia a restricción del crecimiento intrauterino ${ }^{20}$. En pacientes con FEVI < $35 \%$ pueden utilizarse los inhibidores de los receptores de mineralocorticoides, como espironolactona o eplerenona, los cuales se consideran seguros durante la lactancia, pero no así durante el embarazo por sus efectos antiandrogénicos ${ }^{21}$. La digoxina en pacientes con indicación puede usarse, siendo considerada segura tanto en el embarazo como durante la lactancia ${ }^{22}$. No hay suficiente evidencia para recomendar el uso de ivabradina durante el embarazo o la lactancia.

\section{Anticoagulación}

Múltiples factores son la base de que se recomiende la anticoagulación en algunos casos de MCPP; por ejemplo, el conocimiento del embarazo per se como 
una condición protrombótica, la existencia de múltiples reportes de casos de pacientes con MCPP asociados a complicaciones tromboembólicas (enfermedad tromboembólica venosa, trombos intracavitarios específicamente a nivel del VI) y la asociación del tratamiento con bromocriptina con la ocurrencia de eventos trombóticos. La American Heart Association recomienda la anticoagulación en pacientes con MCPP y $\mathrm{FEVI}<30 \%$, mientras que la Sociedad Europea de Cardiología lo hace en pacientes con $\mathrm{FEVI}<35 \%$ hasta aproximadamente la sexta a octava semana del puerperio ${ }^{23}$; además, señalan que debe considerarse la anticoagulación en todas las pacientes que reciben tratamiento con bromocriptina ${ }^{16}$. Con respecto a la elección del agente, debe tenerse en cuenta que la warfarina está contraindicada durante gran parte del embarazo y puede usarse durante la lactancia, mientras que las heparinas de bajo peso molecular son seguras en ambos contextos $^{24}$. No hay estudios disponibles sobre los anticoagulantes orales directos en estas situaciones, razón por la cual no se recomiendan.

\section{Fármacos vasodilatadores}

Tanto la hidralazina como los nitratos de uso oral y parenteral (preferiblemente la nitroglicerina) pueden ser usados con seguridad en las pacientes con MCPP en aquellos casos con hipertensión, disfunción grave del VI o congestión pulmonar sin hipotensión ${ }^{16}$.

\section{Diuréticos}

Como parte del tratamiento para los signos y síntomas de congestión y sobrecarga de volumen pueden usarse los diuréticos de asa, específicamente la furosemida, la cual es segura durante el embarazo y la lactancia. Debe evitarse la sobredosificación y su uso en pacientes sin signos ni síntomas de congestión pulmonar ni sistémica, ya que la deshidratación secundaria a la diuresis excesiva puede disminuir la perfusión placentaria ${ }^{25}$.

\section{Casos complicados y terapia avanzada}

Es importante tomar en cuenta las medidas de las cuales disponemos en la actualidad y sus indicaciones que deben considerarse en los casos que se presentan en emergencias como shock cardiogénico, así como en aquellas pacientes que egresan con disfunción grave del VI. Ante la presencia de shock cardiogénico, dependiendo de las características hemodinámicas con las cuales se presente, puede iniciarse infusión continua de fármacos vasoactivos o inotrópicos, o ambos. Posibles alternativas en estos casos son la dobutamina, el levosimendán y la milrinona. Entre estas drogas, el levosimendán y la milrinona han tenido mejores resultados en cuanto a mejoría de la función sistólica a largo plazo en pacientes con MCPP26,27, mientras que un estudio reportó posibles efectos adversos y evolución no favorable en pacientes que recibieron infusión continua de dobutamina ${ }^{28}$; sin embargo, para los tres fármacos es escasa la evidencia publicada específicamente en MCPP. En casos de inestabilidad hemodinámica que ha sido refractaria al uso de drogas vasoactivas e inotrópicas, el uso de dispositivos de asistencia ventricular izquierda temporal y de larga duración y las terapias de oxigenación extracorpórea han logrado buenos resultados en pacientes con MCPP, por lo que su uso debe considerarse según los algoritmos de manejo de la IC aguda ${ }^{25,29}$. En aquellas pacientes con MCPP y disfunción ventricular grave a largo plazo, el trasplante cardiaco ha tenido una alta tasa de complicaciones ${ }^{30}$.

Con respecto al manejo de las arritmias, debe tenerse en cuenta el riesgo de taquiarritmias ventriculares en aquellas pacientes con disfunción grave del VI. En fase aguda, según las guías para el manejo de la enfermedad cardiovascular durante el embarazo de la SEC publicadas en $2018^{16}$, está recomendada y es segura la cardioversión eléctrica inmediata en las pacientes con inestabilidad hemodinámica, y debe considerarse, de igual forma, como terapia de primera línea en presencia de taquicardia ventricular sostenida monomórfica estable. Opciones para la cardioversión farmacológica son la procainamida, el sotalol, los betabloqueadores y supresión por estimulación ${ }^{16}$. Para la prevención de la muerte súbita a largo plazo en presencia de $\mathrm{FEVI}<35 \%$, tomando en cuenta que una proporción importante de las pacientes presentan recuperación de la función sistólica del VI en los meses próximos a la culminación del embarazo, no se recomienda el implante de un desfibrilador automático implantable (DAl) de forma precoz al egreso ${ }^{16}$. En estos casos se han usado chalecos desfibriladores como terapia puente durante los primeros 6 meses posteriores a la culminación del embarazo, obteniendo buena tasa de choques apropiados y reversión a ritmo sinusal, considerando este un tiempo de espera prudencial para la recuperación de la función sistólica y la reducción del riesgo de muerte súbita para entonces, en caso de requerirlo, implantar un DAl ${ }^{31}$. 


\section{Conclusiones}

Ha ocurrido un incremento en el conocimiento de ciertos aspectos de la MCPP en los últimos años, pero queda mucho por saber respecto a sus mecanismos patogénicos. Si bien la bromocriptina parece ofrecer buenos resultados, es necesaria la realización de más estudios para conocer su verdadero rol en esta condición. En pleno año 2021, el tratamiento de la MCPP sigue estando básicamente orientado al manejo de la IC y sus particularidades inherentes al embarazo y el puerperio, y a la prevención de sus complicaciones. Los estudios experimentales practicados en animales que han aportado datos sobre la participación de aspectos genéticos, inflamación y vías moleculares han expuesto posibles alternativas terapéuticas mediante el uso de fármacos dirigidos hacia el micro-ARN-146, y otros han propuesto el uso de factores de crecimiento endotelial vascular y fármacos como la serelaxina, la perhexilina y la pentoxifilina ${ }^{32}$. Deben realizarse más estudios para evaluar los posibles beneficios y la seguridad de estas alternativas en este grupo de pacientes.

\section{Financiamiento}

El autor no recibió ningún soporte financiero en la elaboración del artículo.

\section{Conflicto de intereses}

Los autores declaran no tener conflictos de intereses.

\section{Responsabilidades éticas}

Protección de personas y animales. Los autores declaran que para esta investigación no se han realizado experimentos en seres humanos ni en animales.

Confidencialidad de los datos. Los autores declaran que en este artículo no aparecen datos de pacientes.

Derecho a la privacidad y consentimiento informado. Los autores declaran que en este artículo no aparecen datos de pacientes.

\section{Bibliografía}

1. Sliwa K, Hilfiker-Kleiner D, Petrie MC, Mebazaa A, Pieske B, Buchmann E, et al Current state of knowledge on aetiology, diagnosis, management, and therapy of peripartum cardiomyopathy: a position statement from the Heart Failure Association of the European Society of Cardiology Working Group on peripartum cardiomyopathy. Eur J Heart Fail. 2010;12:767-78.
2. Hibbard JU, Lindheimer M, Lang RM. A modified definition for peripartum cardiomyopathy and prognosis based on echocardiography. Obstet Gynecol. 1999;94:311-6.

3. Isezuo SA, Abubakar SA. Epidemiologic profile of peripartum cardiomyopathy in a tertiary care hospital. Ethn Dis. 2007;17:228-33.

4. Fett JD, Christie LG, Carraway RD, Murphy JG. Five-year prospective study of the incidence and prognosis of peripartum cardiomyopathy at a single institution. Mayo Clin Proc. 2005;80:1602-6.

5. Krishnamoorthy P, Garg J, Palaniswamy C, Pandey A, Ahmad H, Frishman $\mathrm{WH}$, et al. Epidemiology and outcomes of peripartum cardiomyopathy in the United States: findings from the Nationwide Inpatient Sample. J Cardiovasc Med (Hagerstown). 2016;17:756-61.

6. Goland S, Bitar F, Modi K, Safirstein J, Ro A, Mirocha J, et al. Evaluation of the clinical relevance of baseline left ventricular ejection fraction as a predictor of recovery or persistence of severe dysfunction in women in the United States with peripartum cardiomyopathy. J Card Fail. 2011;17:426-30.

7. Ersbøll A, Johansen M, Damm P, Rasmussen S, Vejlstrup N, Gustafsson F. Peripartum cardiomyopathy in Denmark: a retrospective, population-based study of incidence, management and outcome. Eur J Heart Fail. 2017:19(12):1712-20.

8. Arrigo M, Blet A, Mebazaa A. Bromocriptine for the treatment of peripartum cardiomyopathy: welcome on board. Eur Heart J. 2017;38:2680-2.

9. Bernard $\mathrm{N}$, Jantzem $\mathrm{H}$, Becker $\mathrm{M}$, Pecriaux $\mathrm{C}$, Bénard-Laribière $\mathrm{A}$, Montastruc JL, et al. French Network of Regional Pharmacovigilance Centres. Severe adverse effects of bromocriptine in lactation inhibition: a pharmacovigilance survey. BJOG. 2015;122:1244-51.

10. Hilfiker-Kleiner D, Kaminski K, Podewski $E$, Bonda $T$, Schaefer A, Sliwa K, et al. A cathepsin D-cleaved $16 \mathrm{kDa}$ form of prolactin mediates postpartum cardiomyopathy. Cell. 2007;128:589-600.

11. Hilfiker-Kleiner D, Meyer GP, Schieffer E, Goldmann B, Podewski E, Struman I, et al. Recovery from postpartum cardiomyopathy in 2 patients by blocking prolactin release with bromocriptine. J Am Coll Cardiol. 2007;50:2354-5.

12. Jahns BG, Stein W, Hilfiker-Kleiner D, Pieske B, Emons G. Peripartum cardiomyopathy - a new treatment option by inhibition of prolactin secretion. Am J Obstet Gynecol. 2008;199:e5-6.

13. Sliwa K, Blauwet L, Tibazarwa K, Libhaber E, Smedema JP, Becker A, et al. Evaluation of bromocriptine in the treatment of acute severe peripartum cardiomyopathy: a proof-of-concept pilot study. Circulation. 2010;121:1465-73

14. Yaméogo NB, Kagambèga LJ, Seghda A, Owona A, Kaboré $O$, Kaboré $E$, et al. Bromocriptine in management of peripartum cardiomyopathy: a randomized study on 96 women in Burkina Faso. J Cardiol Clin Res. 2017:5:1098

15. Hilfiker-Kleiner D, Haghikia A, Berliner D, Vogel-Claussen J, Schwab J, Franke A, et al. Bromocriptine for the treatment of peripartum cardiomyopathy: a multicentre randomized study. Eur Heart J. 2017;38:2671-9.

16. Regitz-Zagrosek V, Roos-Hesselink JW, Bauersachs J, Blomström-Lundqvist C, Cífková R, De Bonis M, et al. 2018 ESC Guidelines for the management of cardiovascular diseases during pregnancy. Eur Heart J. 2018:39:3165-241.

17. Davis MB, Arany Z, McNamara DM, Goland S, Elkayam U. Peripartum cardiomyopathy: JACC State-of-the-Art Review. J Am Coll Cardiol. 2020;75:207-21.

18. Schroeter MR, Unsold B, Holke K, Schillinger W. Pro-thrombotic condition in a woman with peripartum cardiomyopathy treated with bromocriptine and an Impella LP 2.5 heart pump. Clin Res Cardiol. 2013;102:155-7.

19. Fala L. Entresto (sacubitril/valsartan): first-in-class angiotensin receptor neprilysin inhibitor FDA approved for heart failure. Am Health Drug Benefits. 2016;9(Spec Feature):78-82.

20. Tanaka K, Tanaka $H$, Kamiya $C$, Katsuragi S, Sawada M, Tsuritani M, et al. Beta-blockers and fetal growth restriction in pregnant women with cardiovascular disease. Circ J. 2016;80:2221-6.

21. Enriquez AD, Economy KE, Tedrow UB. Contemporary management of arrhythmias during pregnancy. Circ Arrhythm Electrophysiol. 2014; 7:961-7.

22. Elkayam U, Goland S, Pieper PG, Silversides CK. High-risk cardiac disease in pregnancy: part II. J Am Coll Cardiol. 2016;68:502-16.

23. Arany Z, Elkayam U. Peripartum cardiomyopathy. Circulation. 2016;133: 1397-409.

24. Briggs GG, Freeman RK, Towers CV, Forinash AB. Drugs in pregnancy and lactation: a reference guide to fetal and neonatal risk. $11^{\text {th }}$ ed. Philadelphia, PA: Wolters Kluwer; 2017.

25. Hilfiker-Kleiner D, Westhoff-Bleck M, Gunter HH, von Kaisenberg CS, Bohnhorst B, Hoeltje M, et al. A management algorithm for acute heart failure in pregnancy. The Hannover experience. Eur Heart J. 2015; 36:769-70.

26. Labbene I, Arrigo M, Tavares M, Hajjej Z, Brandão JL, Tolppanen H, et al. Decongestive effects of levosimendan in cardiogenic shock induced by postpartum cardiomyopathy. Anaesth Crit Care Pain Med. 2017;36:39-42. 
27. Abdel Hamid H, El-Tohamy S. Comparison between milrinone and levosimendan infusion in patients with peripartum cardiomyopathy. Ains Shams Journal of Anesthesiology. 2014;7:114-20.

28. Stapel B, Kohlhaas M, Ricke-Hoch M, Haghikia A, Erschow S, Knuuti J, et al. Low STAT3 expression sensitizes to toxic effects of beta-adrenergic receptor stimulation in peripartum cardiomyopathy. Eur Heart $\mathrm{J}$. 2017:38:349-61.

29. Zimmerman H, Bose R, Smith R, Copeland JG. Treatment of peripartum cardiomyopathy with mechanical assist devices and cardiac transplantation. Ann Thorac Surg. 2010;89:1211-7.
30. Costanzo MR, Dipchand A, Starling R, Anderson A, Chan M, Desai S, et al. The International Society of Heart and Lung Transplantation Guidelines for the care of heart transplant recipients. J Heart Lung Transplant. 2010;29:914-56.

31. Duncker D, Haghikia A, Konig T, Hohmann S, Gutleben KJ, Westenfeld R, et al. Risk for ventricular fibrillation in peripartum cardiomyopathy with severely reduced left ventricular function-value of the wearable cardioverter/defibrillator. Eur J Heart Fail. 2014;16:1331-6.

32. Honigberg MC, Givertz MM. Peripartum cardiomyopathy. BMJ 2019;364:k5287 